Pacific Journal of Mathematics

A SPECTRAL SEQUENCE FOR THE HOMOLOGY OF AN (1) 


\title{
A SPECTRAL SEQUENCE FOR THE HOMOLOGY OF AN INFINITE DELOOPING
}

\author{
HAYNES MILLER
}

Let $E=\left\{E_{n}: n \in Z\right\}$ be a (-1)-connected infinite loop space: i.e., $Q E_{n+1}=E_{n}$ for all $n$, and for $n \geqq 0$ the space $E_{n}$ is $(n-1)$-connected. Then the stable homology of $E$ is

$$
H_{*}(E)=\lim _{\rightarrow} H_{*+n}\left(E_{n}\right)
$$

under the suspension homomorphisms. One also has the $u n$ stable homology $H_{*}\left(E_{0}\right)$, which with $\bmod p$ coefficients carries a Pontrjagin product and an action of the $\bmod p$ Dyer-Lashof algebra $R$.

It is natural to ask how $H_{*}\left(E_{0}\right)$ determines $H_{*}(E)$; and the purpose of this paper is to construct and study the general properties of a spectral sequence whose $E^{2}$-term depends functorially on $H_{*}\left(E_{0}\right)$ as an $R$-Hopf algebra and whose $E^{\infty}$ term is the associated graded module of a natural filtration on $H_{*}(E)$. For simplicity we mainly treat the case $p=2$.

The spectral sequence developed here is probably identical in the stable range with the iterated barconstruction sequence of $D . W$. Anderson. ${ }^{1}$ We give an entirely different construction, however, which makes evaluation of $E^{2}$ easier. In fact, if $\mathscr{A}$ is the category of allowable $R$-Hopf algebras ([4] I or $\S 2$ below) then $E_{s}^{2}$ is the $s$ th derived functor of the functor $\boldsymbol{F}_{p} \otimes_{R} Q(-)$, where $Q(B)$ is the $R$ module of indecomposables of $B \in \mathscr{A}$.

The following heuristic description of the resulting $E^{2}$-term in favorable cases is due to M. Mahowald. Suppose $\pi_{0}\left(E_{0}\right)$ is free-Abelian and $H_{*}\left(E_{0}[1]\right)$ is polynomial, where $E_{0}[1]$ is the connected component of the identity. Then as algebras $H_{*}\left(E_{0}\right)$ is isomorphic to the homology of a product of $\Omega S^{n}$ 's for various $n$. Now $H_{*}\left(\Omega S^{n}\right)$ is an Abelian Hopf algebra and hence has formal iterated deloopings defined inductively by

$$
B^{k} H_{*}\left(\Omega S^{n}\right)=\operatorname{Tor}^{B^{k-1} I I \cdot(\Omega S)^{n}}\left(\boldsymbol{F}_{2}, \boldsymbol{F}_{2}\right) .
$$

Then our $E^{1}$-term is the tensor-product of the corresponding groups $B^{\infty} H_{*}\left(\Omega S^{n}\right)$, and the differential $d^{1}$ is determined by the Dyer-Lashof action on $H_{*}\left(E_{0}\right)$.

In $\S 1$ of this paper we construct the spectral sequence and outline the algebraic identification of its $E^{2}$-term. In $\S 2$ we set up the algebraic background required to complete this identification. We

${ }^{1}$ Added in proof. W. Dwyer points out that our spectral sequence is not identical with Anderson's, since Anderson's $E_{2}$-term depends only on the algebra structure of $H_{*}\left(E_{0}\right)$ while ours depends on the $R$-module structure as well. 
also exploit the fact that $Q(-)$ itself has only one derived functor on an Abelian Hopf algebra to provide a "linear" computation of the $E^{2}$-term. Then in $\S 3$ we set up an analogue of the $\Lambda$-algebra to make this calculation. Finally, we show in $\S 4$ that the action of the Steenrod algebra on $H_{*}\left(E_{0}\right)$ puts very strong restrictions on the differential in the spectral sequence and goes farther than one might expect towards determining the Steenrod action on $H_{*}(E)$.

It is a pleasure to thank Northwestern University for providing the friendly and inspiring atmosphere in which much of this work was done. I am in particular indebted to Mark Mahowald and Stewart Priddy for a great many useful conversations.

1. Construction of the spectral sequence. Let $\mathscr{T}$ be the category of compactly generated Hausdorff spaces with nondegenerate basepoint (i.e., such that the inclusion of the basepoint is a cofibration) and continuous pointed maps. For brevity call an object of $\mathscr{T}$ a space. Clearly $\Omega$ and $\Sigma$ are adjoint functors on $\mathscr{T}$; let $\alpha_{X}: X \rightarrow \Omega \Sigma X$ and $\beta_{Y}: \Sigma \Omega Y \rightarrow Y$ be the usual adjunctions. Let $\mathscr{L}$ be the category of $(-1)$-connected infinite loop spaces. Thus an object $E$ of $\mathscr{L}$ is a sequence $E_{n}, n \in Z$, of spaces such that $E_{n}=\Omega E_{n+1}$ and $E_{n}$ is $(n-1)$-connected for all $n$. A morphism $E^{\prime} \rightarrow E$ is a sequence $f_{n}: E_{n}^{\prime} \rightarrow E_{n}$ of continuous maps such that $f_{n}=\Omega f_{n+1}$ for all $n$. Call an object of $\mathscr{L}$ a spectrum.

Let $\Omega^{\infty}: \mathscr{L} \rightarrow \mathscr{T}$ by $\Omega^{\infty} E=E_{0}$. Let $\Sigma^{\infty}: \mathscr{T} \rightarrow \mathscr{L}$ by

$$
\left(\Sigma^{\infty} X\right)_{n}=\lim _{i \rightarrow \infty} \Omega^{i} \Sigma^{i+n} X .
$$

Then $\Omega^{\infty}$ and $\Sigma^{\infty}$ are adjoint functors [8]; we recall their adjunction morphisms. For $X \in \mathscr{T}, \alpha_{X}^{\infty}: X \rightarrow \Omega^{\infty} \Sigma^{\infty} X$ is the inclusion of the first term in the direct limit (1.1). For $E \in \mathscr{L}_{,}, \beta_{E}^{\infty}: \Sigma^{\infty} \Omega^{\infty} E \rightarrow E$ is the sequence whose $n$th term is the map induced from the system

$$
\Omega^{i} \Sigma^{i+n} E_{0}=\Omega^{i} \Sigma^{i+n} \Omega^{i+n} E_{i+n} \stackrel{\Omega^{i} \beta^{i+n}}{\longrightarrow} \Omega^{i} E_{i+n}=E_{n} .
$$

If $h_{*}$ is a connective homology theory on $\mathscr{T}$ then given $E \in \mathscr{R}$, we can form the unstable homology

$$
h_{*}^{u}(E)=h_{*}\left(\Omega^{\infty} E\right)
$$

and the stable homology

$$
h_{*}(E)=\lim _{n \rightarrow \infty} \Sigma^{-n} \tilde{h}_{*}\left(E_{n}\right) .
$$

Our problem is to relate these groups.

For any spectrum $E$ there exists a space $X$ and a map $i: \Sigma^{\infty} X \rightarrow$ 
$E$ such that $\Omega^{\infty} i$ is a split epimorphism in $\mathscr{T}$. For example, we can take $X=\Omega^{\infty} E$ and $i=\beta_{E}^{\infty}$, since

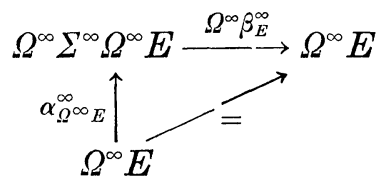

commutes.

Using this observation we can resolve a spectrum $E$ with respect to "space-like" spectra by forming a diagram

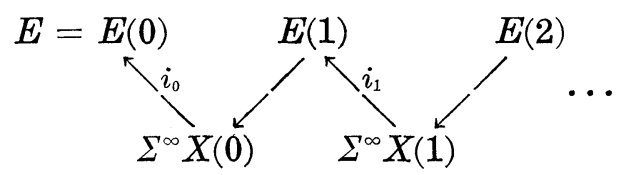

in which (i) $\Omega^{\infty} i_{n}$ is split epi and (ii) the $V$-shaped segments are fibration sequence in $\mathscr{L}$.

Now applying stable homology $h_{*}$ yields a spectral sequence abutting to $h_{*}(E)$.

In case $h_{*}$ is $\bmod p$ singular homology $H_{*}$ for $p$ a prime, we can express $E_{2}$ in this spectral sequence in terms of $H_{*}^{u}(E)$. We shall outline here how this is done, and develop the necessary algebra in the next section.

Apply $\Omega^{\infty}$ to (1.2). The fibration

$$
\Omega^{\infty} E(n+1) \longrightarrow \Omega^{\infty} \Sigma^{\infty} X(n) \stackrel{\Omega^{\infty} i_{n}}{\longrightarrow} \Omega^{\infty} E(n)
$$

is principal, and $\Omega^{\infty} i_{n}$ has a section, so $\Omega^{\infty} \Sigma^{\infty} X(n)$ has the weak homotopy type of the product of fiber and base. Consequently we have a short exact sequence

$$
\boldsymbol{F}_{p} \longrightarrow H_{*}^{u}(E(n+1)) \longrightarrow H_{*}^{u}\left(\Sigma^{\infty} X(n)\right) \longrightarrow H_{*}^{u}(E(n)) \longrightarrow \boldsymbol{F}_{p}
$$

of Hopf algebras over the Dyer-Lashof algebra $R$ (see $\S 2$ below). Splicing these together gives a long exact sequence

$$
\boldsymbol{F}_{p} \longleftarrow H_{*}^{u}(E) \longleftarrow H_{*}^{u}\left(\Sigma^{\infty} X(0)\right) \longleftarrow H_{*}^{u}\left(\Sigma^{\infty} X(1)\right) \longleftarrow \cdots
$$

Now Theorem 4.2 of [4] I implies that for $X \in \mathscr{T}, H_{*}\left(\Sigma^{\infty} X\right)=$ $\widetilde{H}_{*}(X)$ is given in terms of $H_{*}^{u}\left(\Sigma^{\infty} X\right)$ by $\boldsymbol{F}_{p} \otimes_{R} Q H_{*}^{u}\left(\Sigma^{\infty} X\right)$, where $Q$ denotes the Pontrjagin algebra indecomposables. Thus if we regard $H_{*}^{u}\left(\Sigma^{\infty} X\right)$ as a projective object then (1.3) is a projective resolution of $H_{*}^{u}(E)$ and

$$
E_{s}^{2}=L_{s}\left(\boldsymbol{F}_{p} \otimes_{R} Q\right)\left(H_{*}^{u}(E)\right)
$$

is the left derived functor of $\boldsymbol{F}_{p} \otimes_{R} Q(-)$. 
2. The homological algebra of allowable Hopf algebras over the Dyer-Lashof algebra.

\subsection{Hopf algebras. We make the following definitions.}

Let $k$ be a field. Let (Coalg) be the category of nonnegatively graded commutative augumented $k$-coalgebras $C$ such that $C_{0}$ is generated over $k$ by its set

$$
\pi C=\left\{x \in C_{0}: \Delta x=x \otimes x, \varepsilon x=1\right\}
$$

of set-like elements. For brevity call an object of (Coalg) a coalgebra. Given coalgebras $C^{\prime}$ and $C^{\prime \prime}$, the coalgebra $C^{\prime} \otimes C^{\prime \prime}$ is a product in (Coalg). Let (Hopf) be the category of Abelian group objects in (Coalg). Call an object of (Hopf) a Hopf algebra.

For a Hopf algebra $H, \pi H$ has a natural Abelian group structure for which the identity element is the identity element 1 of $H$. Let $H[1]$ be the component of $H$ containing 1: i.e., $H[1]=k \square_{H_{0}} H$. Then the natural map $k[\pi H] \otimes H[1] \rightarrow H$ is an isomorphism. It follows that (Hopf) splits as the product of the category (Ab) Abelian groups and the category (c Hopf) of connected Hopf algebras. By a theorem of J. C. Moore (unpublished, but see [5]), (c Hopf) is Abelian; so (Hopf) is Abelian. The (co) product is the tensor-product.

Let $Z:$ (Coalg) $\rightarrow$ (Hopf) be the "free Abelian group" functor, left adjoint to the forgetful functor; see [4] p. 23 for details. This adjoint pair induces a projective class [6] in (Hopf), so the left derived functors $L_{s} F$ of an additive functor $F$ on (Hopf) are defined.

For example, let $Q:$ (Hopf $) \rightarrow(k-\bmod )$ be the module of indecomposables; thus $Q H=\operatorname{Tor}_{1}^{I I}(k, k)$. There is a six-term exact sequence

$$
0 \longrightarrow Q_{1} H^{\prime} \longrightarrow Q_{1} H \longrightarrow Q_{1} H^{\prime \prime}
$$$$
Q H^{\prime}
$$$$
Q H \longrightarrow Q H^{\prime \prime}
$$

depending functorially on the short exact sequence $k \rightarrow H^{\prime} \rightarrow H \rightarrow$ $H^{\prime \prime} \rightarrow k$ in (Hopf). Here $Q_{1} H=Q \operatorname{Tor}_{2}^{H}(k, k)$ in terms of the natural algebra structure on $\operatorname{Tor}_{*}^{H}(k, k)$. To see this first let $A$ be an Abelian group. Let $E$ denote the exterior algebra over $k$ and let $\Gamma$ denote the divided polynomial algebra over $k$. Then as algebras

$$
\operatorname{Tor}^{k[A]}(k, k)=E(\Sigma(k \otimes A)) \otimes \Gamma\left(\Sigma^{2}(k * A)\right)
$$

where $\Sigma$ means suspension and where $\otimes$ and $*$ are the tensor and torsion products over $Z$. Thus $Q k[A]=k \otimes A$ and $Q_{1} k[A]=k * A$, and (2.1.1) is the long exact sequence of the derived functors of $k \otimes-$. In the connected case (2.1.1) is proved in [9]. The general case follows by additivity.

Now by standard techniques we have 
Proposition 2.1.2. On (Hopf), $L_{0} Q=Q, L_{1} Q=Q_{1}$, and $L_{s} Q=0$ for $s>1$.

2.2. The Dyer-Lashof algebra. For simplicity we fix $p=2$. We recall some notation from [4].

Let $R(-\infty)$ be the bigraded algebra with generators $Q^{i}, i \geqq 0$, $\left\|Q^{i}\right\|=(1, i)$, and "Adem" relations

$$
Q^{i} Q^{j}=\sum_{t}\left(\begin{array}{c}
t-j-1 \\
2 t-i
\end{array}\right) Q^{i+j-t} Q^{t}, \quad i>2 j .
$$

$R(-\infty)$ is thus isomorphic to the opposite of the $\Lambda$-algebra [2]. We will often neglect the first gradation to obtain a graded algebra. Let $s$ be a nonnegative integer and let $I$ be a sequence of $s$ nonnegative integers $i_{1}, \cdots, i_{s} . \quad I$ is allowable iff $i_{t} \leqq 2 i_{t+1}$ for $1 \leqq t<s$. Let $Q^{I}=Q^{i_{1}} \cdots Q^{i_{s}} \in R(-\infty)$. Then $\left\{Q^{I}: I\right.$ is allowable $\}$ is a basis for $R(-\infty)$.

The excess of an allowable sequence $I$ of length $s$ is $e(I)=i_{1}$ $\left(i_{2}+\cdots+i_{s}\right)$. The subspace spanned by $\left\{Q^{I}: e(I)<n\right\}$ is an ideal $B(n) \subset R(-\infty)$. Let $R(n)=R(-\infty) / B(n)$. Then $R=R(0)$ is the DyerLashof algebra.

An $R(-\infty)$-module $M$ is $n$-allowable iff $Q^{i} x=0$ for all $x \in M$ and all $i<|x|+n$. Thus for any nonnegatively graded $n$-allowable $R(-\infty)$-module, the $R(-\infty)$-action factors through an $R(n)$-action. Write $\left(a_{n} R\right.$-mod) for the Abelian category $n$-allowable $R(-\infty)$-modules.

For $n \in Z$, the inclusion $\left(a_{n} R\right.$-mod $) \rightarrow(R(-\infty)$-mod) has a leftadjoint $a_{n}$ such that

$$
a_{n} M=M /\left\{Q^{i} x: i<|x|+n, x \in M\right\} .
$$

This adjoint pair induces a projective class in $\left(a_{n} R\right.$-mod). Let $\operatorname{Untor}_{*}^{R}\left(\boldsymbol{F}_{2},-\right)$ denote the resulting derived functor of

$$
\boldsymbol{F}_{2} \otimes_{R}-:\left(a_{1} R-\bmod \right) \longrightarrow\left(\boldsymbol{F}_{2}-\bmod \right) \text {. }
$$

2.3. Hopf algebras over the Dyer-Lashof algebra. The DyerLashof algebra admits a (noncommutative) Hopf algebra structure with commutative diagonal such that $\Delta Q^{n}=\sum_{i+j=n} Q^{i} \otimes Q^{j}$. Hence one has a internal tensor-product on $\left(a_{0} R\right.$-mod) and so one can define the category ( $a R$-Coalg) of allowable $R$-coalgebras and ( $a R$-Hopf) of allowable $R$-Hopf algebras. (An $R$-Hopf algebra $H$ is allowable if it is 0 -allowable qua $R$-module and is such that $Q^{|x|} x=x^{2}$ for all $x \in H$.) It is easy to see that ( $a R$-Hopf) is Abelian.

In [4] I $\S 2$ May constructs adjoint functors 


$$
\text { (Coalg) } \stackrel{E}{\rightleftarrows}(a R \text {-Coalg }) \stackrel{Z}{\rightleftarrows}(a R \text {-Hopf })
$$

where the unnamed arrows are forgetful functors. $E$ is such that $J E C=a_{0}(R \otimes J C)$, and $Z$ (which May calls $G W$ ) covers $Z:($ Coalg) $\rightarrow$ (Hopf). Give ( $a R$-Hopf) the projective class induced by $Z E$.

By [4] I Theorem 4.2, $H_{*}^{u}\left(\Sigma^{\infty} X\right) \cong Z E H_{*}(X) \in(a R$-Hopf) naturally in $X \in \mathscr{T}$. Thus (1.3) is indeed a projective resolution; note in particular that it is split as a sequence of coalgebras. Thus if $\boldsymbol{Q}$ : $(a R$-Hopf $) \rightarrow\left(\boldsymbol{F}_{2}\right.$-mod $)$ by $\boldsymbol{Q} H=\boldsymbol{F}_{2} \otimes_{R} Q H$, then in our spectral sequence,

$$
E_{s}^{2}=L_{s} \boldsymbol{Q}\left(H_{*}^{u}(E)\right) \text {. }
$$

To compute $L_{s} Q$ we first study $Q:(a R$-Hopf $) \rightarrow\left(a_{1} R\right.$-mod $)$. Let $H \leftarrow P_{*}$ be a projective resolution in (aR-Hopf). Clearly $P_{*}$ is a projective resolution of $H$ when regarded in (Hopf). Hence for $H \in$ (aR-Hopf),

$$
L_{1} Q(H)=Q \operatorname{Tor}_{2}^{H}\left(\boldsymbol{F}_{2}, \boldsymbol{F}_{2}\right)
$$

as an $\boldsymbol{F}_{2}$-module and $L_{t} Q(H)=0$ for $t>1$.

It is easy to see that $Q:(a R$-Hopf $) \rightarrow\left(a_{1} R\right.$-mod $)$ carries projectives to projectives. One thus has a Grothendieck spectral sequence [6]

$$
E_{s, t}^{2}=\operatorname{Untor}_{s}^{R}\left(\boldsymbol{F}_{2}, L_{t} Q(H)\right) \underset{s}{\Longrightarrow} L_{s+t} \boldsymbol{Q}(H) .
$$

Since $L_{t} Q=0$ for $t>1$ this spectral sequence degenerates to a long exact sequence

$$
\begin{aligned}
0 \longleftarrow \operatorname{Untor}_{1}^{R}\left(\boldsymbol{F}_{2}, Q H\right) \longleftarrow L_{1} \boldsymbol{Q}(H) & \longleftarrow \operatorname{Untor}_{0}^{R}\left(\boldsymbol{F}_{2}, Q_{1} H\right) \\
& \stackrel{\partial}{\longleftarrow} \operatorname{Untor}_{2}^{R}\left(\boldsymbol{F}_{2}, Q H\right) \longleftarrow \ldots .
\end{aligned}
$$

In particular, if $Q_{1} H=0$ - for example, if $\pi H$ is free of 2 -torsion and $H[1]$ is a symmetric algebra - then

$$
L_{s} \boldsymbol{Q}(H)=\operatorname{Untor}_{s}^{R}\left(\boldsymbol{F}_{2}, Q H\right)
$$

for all $s$. Thus in this case $L_{s} \boldsymbol{Q}(H)$ is determined by the action of $R$ on $Q H$ alone.

REMARK 2.3.5. Of course, this $E_{2}$-term may also be obtained directly, without introducing $\boldsymbol{Q}$, by observing that

$$
0 \longleftarrow Q H_{*}^{u}(E) \longleftarrow Q H_{*}^{u}\left(\Sigma^{\infty} X(0)\right) \longleftarrow Q H_{*}^{u}\left(\Sigma^{\infty} X(1)\right) \longleftarrow \cdots
$$

is a projective resolution in $\left(a_{1} R\right.$-mod) which yields $E^{1}$ on application of the functor $\boldsymbol{F}_{z} \otimes_{R}$. 
2.4. Remark on the Adams spectral sequence. The procedure of this section is equally applicable to the study of the $E_{2}$-term of Bousfield and Kan's mod $p$ unstable Adams spectral sequence [2]. They prove that

$$
E_{2}^{s}(X)=R^{s} P\left(H_{*}(X)\right) .
$$

Here $\boldsymbol{P}$ : (unstable $A$-coalgebras) $\rightarrow\left(\boldsymbol{F}_{p}\right.$-mod) is the functor $\boldsymbol{F}_{p} \square_{A} P(-)$ where $A$ is the $\bmod p$ dual Steenrod algebra and $P(-)$ is the module of primitives. Then $P(C)$ is a 1-unstable $A$-comodule, in the sense (for $p=2$ ) that $x S q^{n}=0$ for all $x \in C$ and all $n \geqq 1 / 2|x|$. As such, $P$ carries injectives to injectives, and there results a Grothendieck spectral sequence

$$
E_{2}^{s, t}=\text { Uncotor }_{A}^{s}\left(\boldsymbol{F}_{p}, R^{t} P(C)\right) \underset{s}{\Longrightarrow} R^{s+t} \boldsymbol{P}(C)
$$

in which Uncotor ${ }_{A}^{s}\left(\boldsymbol{F}_{p},-\right)$ is the obvious right derived functor of $\boldsymbol{F}_{p} \square_{A}-:\left(u_{1} A\right.$-comod $) \rightarrow\left(\boldsymbol{F}_{p}\right.$-mod $)$.

This situation is in several ways more complicated than the DyerLashof case. First, the category of unstable $A$-coalgebras is not additive, so one must use the theory of nonabelian derived functors to define $R^{s} P$ and $R^{s} P$. Furthermore, $R^{s} P$ can be nonzero for all $s$. Bousfield and Curtis consider the case when $R^{s} P=0$ for $s>1$, and [1] is in part an analysis of the spectral sequence (2.4.1) in this "nice" case.

Bousfield and Curtis construct a complex $\Lambda(M)$ such that

$$
\operatorname{Uncotor}_{A}\left(\boldsymbol{F}_{2}, M\right)=H(\Lambda(M)) \text {. }
$$

$\Lambda(M)$ is closely related to the usual unstable $\Lambda$-algebra for $M$. Our next step here is establish an analogous complex for a 1-allowable $R(-\infty)$-module. We remark that the methods of $\S 3$ carry over to the Steenrod algebra case and give another derivation of the properties of $\Lambda(M)$.

3. A 4 -algebra for allowable $R$-modules. We now address the problem of computing $\operatorname{Untor}^{R}\left(\boldsymbol{F}_{2}, M\right)$. As noted by S. Priddy in [10], Example 9.4, the algebra $R(-\infty)$ has a "Koszul resolution." We note here that this machinery behaves well with respect to the allowability condition, and study the resulting complex.

3.1. The off-diagonal homology of the bar-construction. Let

$$
R=R(-\infty) \otimes-:\left(\boldsymbol{F}_{2}-\bmod \right) \longrightarrow(R(-\infty)-\bmod )
$$

and

$$
U=a_{1}(R(-\infty) \otimes-):\left(\boldsymbol{F}_{2}-\mathrm{mod}\right) \longrightarrow\left(a_{1} R-\bmod \right)
$$


be left adjoint to the forgetful functors. The resulting cotriples on $\left(R(-\infty)\right.$-mod) and $\left(a_{1} R\right.$-mod) give rise to standard complexes $B_{R}(M)$ and $B_{U}(M)$. Here $B_{R}(M)$ is the usual (reduced) unnormalized bar construction with respect to $R(-\infty)$. Similarly, $B_{U}(M)_{s}=U^{s}(M)$. The natural surjection $R(M) \rightarrow U(M)$ induces a surjection of the corresponding bar constructions.

Given a basis $X$ for $M \in(R(-\infty)$-mod),

$$
\left\{Q^{I_{1}} \otimes \cdots \otimes Q^{I_{s}} \otimes x: I_{j} \quad \text { is allowable, } \quad x \in X\right\}
$$

is a basis for $B_{R}(M)_{s}$. If $M \in\left(a_{1} R\right.$-mod) then a basis for $B_{U}(M)_{s}$ is given by (the image of) the subset of $Q^{I_{1}} \otimes \cdots \otimes Q^{I_{s}} \otimes x$ in (3.1.1) such that in addition

$$
e\left(I_{j}\right)>\left|I_{j+1}\right|+\cdots+\left|I_{s}\right|+|x|
$$

for $1 \leqq i \leqq s$. These are the "monomial" bases.

Let $S_{n} \in\left(a_{1} R\right.$-mod) have $\left(S_{n}\right)_{n}=\boldsymbol{F}_{2}$ (with generator $\left.x\right)$ and $\left(S_{n}\right)_{j}=0$ for $j \neq n$. Then the bigrading on $R(-\infty)$ induces a trigrading on $B_{R}\left(S_{n}\right)$ and on $B_{U}\left(S_{n}\right)$ with $\left(B_{R}\left(S_{n}\right)\right)_{s, t, u}$ generated by those $Q^{I_{1}} \otimes \cdots \otimes$ $Q^{I_{s}} \otimes x$ such that $\sum l\left(I_{j}\right)=t$ and $\sum\left|I_{j}\right|+n=u$. Clearly $B_{R}\left(S_{n}\right)_{s, t, u}=$ 0 for $s>t$. The differential $d$ preserves $t$ and $u$ and decreases $s$ by 1. We now have:

Proposition 3.1.2. There is no homology in $B_{R}\left(S_{n}\right)$ or in $B_{U}\left(S_{n}\right)$ off the main diagonal - i.e., for $s \neq t$.

For $B_{R}\left(S_{n}\right)$ this is [10] Theorem 5.3. The assertion follows for $B_{U}\left(S_{n}\right)$ upon checking that the chain-homotopy constructed by Priddy sends $B_{U}\left(S_{n}\right)$ into itself.

To compute the diagonal homology, we pass to the dual, still following [10].

3.2. The dual of the Dyer-Lashof algebra. We recall from [7] and [4] the structure of the dual $\hat{R}$ of the Dyer-Lashof algebra. As a commutative algebra

$$
\hat{R}=\prod_{k \geqq 0} \hat{R}[k]
$$

where $\hat{R}[k]=\boldsymbol{F}_{2}\left[\xi_{1, k}, \cdots, \xi_{k, k}\right]$ for $k>0$, and $\hat{R}[0]=\boldsymbol{F}_{2}$. Here $\xi_{i, k}$ is dual (with respect to the allowable basis) to $Q^{I_{i, k}}$, where $I_{i, k}$ is the length $k$ sequence

$$
\begin{aligned}
& I_{0, k}=(0, \cdots, 0) \\
& I_{i, k}=\left(2^{k-i-1}\left(2^{i}-1\right), \cdots,\left(2^{i}-1\right), 2^{i-1}, \cdots, 1\right) \text { for } 0<i<k \\
& I_{k, k}=\left(2^{k-1}, \cdots, 1\right) .
\end{aligned}
$$


Note that $e\left(Q I_{i, k}\right)=0$ unless $i=k>0$, when $e\left(Q I_{k, k}\right)=1 . \quad \xi_{0, k}$ is the identity element in $\hat{R}[k] . \quad \hat{R}$ is bigraded by $\left\|\xi_{i, k}\right\|=\left(k, 2^{k-i}\left(2^{i}-1\right)\right)$. Let $\Lambda$ be a sequence of the form $\left(k ; i_{1}, \cdots, i_{k}\right)$ where $k \geqq 0$ and $1 \leqq$ $i_{j} \leqq k$; then define $\xi^{\Lambda}=\xi_{i_{1}, k} \cdots \xi_{i_{k}, k}$. Write $l(\Lambda)=k$.

$R$ is not a Hopf algebra, because $R_{0}=\boldsymbol{F}_{2}\left[Q^{0}\right]$ is not of finite type. However, the product in $R$ induces a multiplicative unitary coproduct $\Delta$ on $\hat{R}_{+}$, the positive dimensional submodule of $\hat{R}$, such that

$$
\Delta \xi_{j, l}=\sum_{h, 2}\left(\xi_{k-i, k-i}^{2^{h}-1} \xi_{j-k, k-i}\right)^{2^{i-h}} \otimes \xi_{h, i} \cdot
$$

Also the unit in $R$ dualizes to $\varepsilon: \hat{R} \rightarrow \boldsymbol{F}_{2}$ by $\varepsilon\left(\xi_{0,0}\right)=1$ and $\varepsilon(x)=0$ otherwise.

Let $J(n)$ be the submodule of $\hat{R}$ generated by $\left\{\xi^{A}: i_{k} \geqq n\right.$ where $l(\Lambda)=k\} ; J(n)$ is the ideal generated by $\left\{\hat{\xi}_{k, k}^{n}: k \geqq 1\right\} . J(n)$ is then the annihilator of $B(n) \subset R$.

A 1-allowable comodule over $R$ is a nonnegatively graded $\boldsymbol{F}_{2}$ module together with a map $\psi: N \rightarrow \hat{U}(N)$, where

$$
\hat{U}(N)=\bigoplus_{n \geq 0} J(n+1) \otimes N_{n} \subset \hat{R} \otimes N,
$$

such that $(\Delta \otimes N) \psi=(\hat{R} \otimes \psi) \psi$ and $(\varepsilon \otimes N) \psi=i d$. Notice that since $J(n+1)_{0}=0$ for $n \geqq 0, \Delta \otimes N$ is defined on $\hat{U}(N)$.

Let ( $a_{1} \hat{R}$-comod) denote the resulting category. It is clearly Abelian, and there are functors

$$
\left(\boldsymbol{F}_{2} \text {-mod }\right) \stackrel{\hat{U}}{\rightleftarrows}\left(a_{1} \hat{R} \text {-comod }\right)
$$

in which the unmarked arrow is forgetful and $\hat{U}$ is its right adjoint. This adjoint pair yields an injective class in ( $a_{1} R$-comod) and a standard complex $\Omega_{\hat{V}}(N)$. A basis $Y$ for $N$ determines a basis for $\Omega_{\hat{i}}(N)^{s}$, namely,

$$
\begin{array}{r}
\left\{\xi^{\Lambda_{1}} \otimes \cdots \otimes \xi^{\Lambda_{s}} \otimes y: \xi^{\Lambda_{j}} \in J\left(\left|\Lambda_{j+1}\right|+\cdots+\left|\Lambda_{s}\right|+|y|\right)\right. \\
\text { for all } 1 \leqq j \leqq s \text { and } y \in Y\}
\end{array}
$$

If $M$ is a 1-allowable $R$-module then $\hat{M}$ is a 1-allowable $R$-comodule and there is a natural isomorphism of complexes

$$
B_{U}(M)^{\wedge}=\Omega_{\hat{U}}(\hat{M}) .
$$

Thus if $M$ is of finite type,

$$
\operatorname{Untor}^{R}\left(\boldsymbol{F}_{2}, M\right)^{\wedge}=H\left(\Omega_{\hat{U}}(\hat{M})\right) \text {. }
$$

3.3. The diagonal homology of the cobar construction. We must therefore compute $H^{s, s}\left(\Omega_{\hat{U}}\left(\hat{S}_{n}\right)\right)$. From (3.2.2), $\Omega_{\hat{U}}\left({\widehat{S_{n}}}^{s, s}\right.$ has basis 
(3.3.1) $\quad\left\{\xi_{11}^{i_{1}} \otimes \cdots \otimes \xi_{11}^{i_{s}} \otimes y: i_{j}>i_{j+1}+\cdots+i_{s}+n\right.$ for $\left.1 \leqq j \leqq s\right\} ，$ where $y$ generates $\hat{S}_{n}$. These elements are of course all cycles, since $\Omega_{\hat{U}}\left(\hat{S}_{n}\right)^{s+1, s}=0$. This also follows from the equations

$$
\Delta \xi_{11}=\xi_{11} \otimes \xi_{00}+\xi_{00} \otimes \xi_{11}
$$

and $\xi_{00} \xi_{11}=0$, since then

$$
\Delta \xi_{11}^{i}=\xi_{11}^{i} \otimes \xi_{00}+\xi_{00} \otimes \xi_{11}^{i} .
$$

To compute the boundaries in $\Omega_{\hat{V}}\left(\widehat{S}_{n}\right)^{s, s}$ note that $\Omega_{\hat{V}}\left(\hat{S}_{n}\right)^{s-1, s}$ has basis

$$
\begin{aligned}
& \left\{\xi_{11}^{i_{1}} \otimes \cdots \otimes \xi_{11}^{i_{k-1}} \otimes \xi_{12}^{i} \xi_{22}^{i_{k}} \otimes \xi_{11}^{i_{k+1}} \otimes \cdots \otimes \xi_{11}^{i_{s-1}} \otimes y:\right. \\
& \left.i \geqq 0 \text { and } i_{j}>i_{j+1}+\cdots+i_{s-1}+n \text { for } 1 \leqq j \leqq s-1\right\}
\end{aligned}
$$

Now

$$
\begin{aligned}
& \Delta \xi_{12}=\xi_{12} \otimes \xi_{00}+\xi_{11} \otimes \xi_{11}+\xi_{11}^{2} \otimes \xi_{01}+\xi_{00} \otimes \xi_{12} \\
& \Delta \xi_{22}=\xi_{22} \otimes \xi_{00}+\xi_{00} \otimes \xi_{22}+\xi_{11}^{2} \otimes \xi_{11}
\end{aligned}
$$

so the reduced diagonal of $\xi_{12}^{i} \xi_{22}^{j}$ is

$$
\sum_{t=0}^{i}\left(\begin{array}{l}
i \\
t
\end{array}\right) \xi_{11}^{2 i+2 j-t} \otimes \xi_{11}^{j+t}
$$

and this obviously determines the differential of an element of (3.3.3). To describe the resulting homology, let $L$ be the associative algebra on symbols $\sigma_{i+1}, i \geqq 0$, of bidegree $(1, i)$, subject to relations

$$
\sum_{t=0}^{i}\left(\begin{array}{l}
i \\
t
\end{array}\right) \sigma_{2 i+2 j-t+1} \sigma_{j+t+1}=0
$$

for $i, j \geqq 0$. Then $H\left(\Omega_{\hat{i}}\left(\widehat{S}_{n}\right)\right)$ maps isomorphically to the sub $\boldsymbol{F}_{2}$-module of $L$ spanned by

$$
\left\{\sigma_{i_{1}+1} \cdots \sigma_{i_{s}+1}: i_{j}>i_{j+1}+\cdots+i_{s}+n \text { for } 1 \leqq j \leqq s\right\}
$$

by sending $\xi_{11}^{i_{1}} \otimes \cdots \otimes \xi_{11}^{i_{s}} \otimes y$ to $\sigma_{i_{1}+1} \cdots \sigma_{i_{s}+1}$.

LeMmA 3.3.8. (a) Let $I=\left(i_{1}, \cdots, i_{s}\right)$ be a sequence of positive integers such that

$$
i_{j} \geqq i_{j+1}+\cdots+i_{s} \quad \text { for } 1 \leqq j<s .
$$

Then in $L, \sigma_{I+1}=\sigma_{i_{1}+1} \cdots \sigma_{i_{s}+1}$ is a linear combination of $\sigma_{J}$ 's with $J$ admissible.

(b) $\left\{\sigma_{J}: J\right.$ is admissible $\}$ is linearly independent in $L$. 
Here "admissible" carries its usual meaning: $J$ is admissible iff $j_{k} \geqq 2 j_{k+1}$ for all $k$.

Proof. (a) Let $0 \leqq b \leqq a \leqq 2 b$. Then with the substitutions $j=$ $a-b, i=2 b-a, t=2 b-a-s$, (3.3.7) yields

$$
\sigma_{a+1} \sigma_{b+1}=\sum_{s=0}^{2 b-a}\left(\begin{array}{c}
2 b-a \\
s
\end{array}\right) \sigma_{a+s+1} \sigma_{b-s+1} .
$$

The condition (3.3.9) guarantees the applicability of this relation to $\sigma_{I+1}$ unless $I+1$ is admissible. The reader may check that (3.3.9) is satisfied by each term of the result. If we define the moment of $I+1$ as

$$
m(I+1)=\sum_{j=1}^{s} j\left(i_{j}+1\right)
$$

then each term of the result has strictly smaller moment. This completes the proof, since moment is positive.

(b) If we forget the first gradation, then $L$ maps multiplicatively to the mod 2 Steenrod algebra $A$ by sending $\sigma_{\imath+1}$ to $S q^{i+1}$ for $i \geqq 0$. To see this let $\delta: A \rightarrow A$ be the derivation sending $S q^{i+1}$ to $S q^{i}$. Then (3.3.7) maps to Leibnitz formula for $\delta^{i}\left(S q^{2 i+2 j+1} S q^{i+j+1}\right)$, which is zero as required. Since $\left\{S q^{I}: I\right.$ is admissible $\}$ is a basis for $A$, the result follows.

We have proved

Proposition 3.3.11. Let $n \geqq 0$. Then

$$
\operatorname{Untor}_{s}^{R}\left(\boldsymbol{F}_{2}, S_{n}\right)^{\wedge}=H_{s}\left(B_{U}\left(S_{n}\right)\right)^{\wedge}=H^{s}\left(\Omega_{\hat{l}}\left(\hat{S}_{n}\right)\right)=L^{s}(n)
$$

where $L^{s}(n)$ is the $\boldsymbol{F}_{2}$-module with basis

$$
\left\{\sigma_{I}: I \text { admissible, } l(I)=s, i_{s}>n+1\right\} \text {. }
$$

ExAMPLE 3.3.12. Let $H$ be the integral Eilenberg-MacLane spectrum. Then $\Omega^{\infty} H=\boldsymbol{Z}$ as a discrete $H$-space, so $H_{*}^{u}(H)=\boldsymbol{F}_{2}[\boldsymbol{Z}]$. Thus (2.3.4) applies, and since $Q \boldsymbol{F}_{2}[\boldsymbol{Z}]$ is spanned by $x=[1]-[0]$, our $E^{2}$-term is $\operatorname{Untor}_{*}^{R}\left(\boldsymbol{F}_{2}, \boldsymbol{F}_{2}\right)=L^{*}(0)^{\wedge}$. Now $H_{*}(H)^{\wedge}=A / A S q^{1}$, and we have just seen that $L^{*}(0)$ is additively isomorphic to $A / A S q^{1}$ under the correspondence $\sigma_{i+1} \leftrightarrow S q^{i+1}, i \geqq 1$. The spectral sequence there fore collapses in this case. The example of the mod 2 EilenbergMacLane spectrum may be handled using (2.3.3), and this provides an upper bound (which turns out to be sharp) on the size of the Steenrod algebra, derived entirely from the Dyer-Lashof algebra. We shall study the behavior of the Steenrod action in the spectral sequence in more detail in $\S 4$. 
We now define a complex $L(N)$, natural in the 1-allowable $\hat{R}$ comodule $N$, such that

$$
H\left(\Omega_{\hat{\imath}}(N)\right)=H(L(N)) .
$$

As an $\boldsymbol{F}_{2}$-module,

$$
L(N)=\bigoplus_{n=0} L(n) \otimes N_{n}
$$

To define the differential, let $\pi_{1}: \hat{R} \rightarrow \hat{R}[1]$ be the projection; then

$$
\left(\pi_{1} \otimes 1\right) \psi(y)=\sum_{i>0} \xi_{11}^{i} \otimes y Q^{i}
$$

defines a right $R$-action on $N$. Let

$$
d\left(\sigma_{I} \otimes y\right)=\sum_{i>0} \sigma_{I, i+1} \otimes y Q^{i} .
$$

Now the relations in $L$ precisely guarantee that $d^{2}=0$. There is a natural map of complexes

$$
\theta: \Omega_{\hat{U}}(N) \longrightarrow L(N)
$$

sending $\xi^{\Lambda_{1}} \otimes \cdots \otimes \xi^{1_{s}} \otimes y$ to zero unless $l\left(\Lambda_{j}\right)=1$ for all $j$, and sending

$$
\xi_{11}^{i_{1}} \otimes \cdots \otimes \xi_{11}^{i_{s}} \otimes y \longmapsto \sigma_{i_{1}+1} \cdots \sigma_{i_{s}+1} \otimes y .
$$

Filter $N$ by $F_{n} N=\bigoplus_{i \leqq n} N^{i} . \quad F_{n} N$ is a sub 1-allowable $\hat{R}$-comodule, and $E_{n}^{0} N$ is a sum of copies of $\hat{S}_{n}$. Thus in the resulting spectral sequence, $E^{1}(\theta)$ is an isomorphism by the above work. Thus $\theta$ is a homology isomorphism, and we have proved

THEOREM 3.3.16. There is an isomorphism

$$
\operatorname{Untor}^{R}\left(\boldsymbol{F}_{2}, M\right)^{\wedge} \cong H(L(\widehat{M}))
$$

natural the 1-allowable $R(-\infty)$-module $M$ of finite type.

\section{The Steenrod action.}

4.1. The Nishida action. Let $A^{0}$ be the opposite of the mod 2 Steenrod algebra, graded nonpositively. A left $A^{0}$-module $M$ is unstable iff $S q^{n} x=0$ whenever $2 n>|x|$. Write $\left(u A^{0}\right.$-mod) for the resulting Abelian category. The diagonal $A^{0}$-action provides it with an internal tensor-product. A coalgebra $C$ in $\left(u A^{0}\right.$-mod) is unstable provided that $\xi x=S q^{n} x$ for all $x \in C_{2 n}$, where $\xi$ is the Verschiebung.

If $M$ is an unstable $A^{0}$-module, give $R \otimes M$ the unstable $A^{0}$-module structure determined inductively on length in $R$ by the Nishida relation 


$$
S q^{n}\left(Q^{r} x\right)=\sum_{t}\left(\begin{array}{c}
r-n \\
n-2 t
\end{array}\right) Q^{r-n+t} S q^{t} x
$$

An $n$-allowable $R(-\infty)$-module structure on $M$ (for $n \geqq 0$ ) is an $A^{0} R$ structure iff $R \otimes M \rightarrow M$ is an $A^{0}$-module map. The resulting category $\left(u a_{n} A^{0} R\right.$-mod) is Abelian.

Putting this together with $\S 2.3$, we have the category $\left(u a A^{0} R\right.$ Hopf) of allowable $R$-Hopf algebras with compatible unstable $A^{0}$-action. ( $u a A^{\circ} R$-Hopf) is Abelian, and the functors $Z, E$, and $\boldsymbol{Q}$ of $\S 2.3$ lift; for example, $Q$ : $\left(u a A^{0} R\right.$-Hopf $) \rightarrow\left(u A^{0}\right.$-mod $)$ additively. Then [4] I Theorem 4.2 asserts further that $H_{*}^{u}\left(\Sigma^{\infty} X\right) \cong Z E H_{*}(X)$ in $\left(u a A^{0} R\right.$ Hopf). Thus the spectral sequence is defined in $\left(u A^{0}\right.$-mod), and $E_{s}^{2}=$ $L_{s} \boldsymbol{Q}\left(H_{*}^{u}(X)\right)$ in $\left(u A^{0}\right.$-mod).

Similarly, for $M \in\left(u a_{1} A^{0} R\right.$-mod $)$, $\operatorname{Untor}_{s}^{R}\left(\boldsymbol{F}_{2}, M\right) \in\left(u A^{0}\right.$-mod), and the exact sequence (2.3.3) is defined in $\left(u A^{0}\right.$-mod). When $M$ is of finite type we can express this action in terms of the complex $L(\widehat{M})$ introduced in $\S 3.3$. Give $\hat{M}$ the contragredient left $A$-action. There is then an $A$-action on $\hat{U}(\hat{M})$ for which $\psi: \hat{M} \rightarrow \hat{U}(\widehat{M})$ is $A$-linear. We need only part of this action, namely,

$$
S q^{n}\left(\xi_{11}^{r} \otimes x\right)=\sum_{t}\left(\begin{array}{c}
r-t \\
n-2 t
\end{array}\right) \xi_{11}^{n+r-t} \otimes S q^{t} x
$$

This formula follows by an easy dualization from (4.1.1). Thus one may inductively define an $A$-module structure on $L(\hat{M})$ : if it is known on $L^{s-1}(\hat{M})$, let

$$
S q^{n}\left(\sigma_{r+1} w\right)=\sum_{t}\left(\begin{array}{c}
r-t \\
n-2 t
\end{array}\right) \sigma_{n+r-t+1} S q^{t} w
$$

for $w \in L^{s-1}(\hat{M})$. Then $L(\hat{M})$ is a differential-graded object in $(u A$-mod), the category of left $A$-modules for which $S q^{n} x=0$ whenever $n>|x|$, and

$$
H(L(\hat{M})) \cong \operatorname{Untor}^{R}\left(\boldsymbol{F}_{2}, M\right)^{\wedge}
$$

as $A$-modules.

EXAMPLE 4.1.4. By comparing (4.1.3) with the Adem relations in the Steenrod algebra, we have

$$
L^{n}\left(\widehat{S}_{0}\right)=L^{n}(0) \cong E_{0}^{n}\left(A / A S q^{1}\right)
$$

as $A$-modules, where $A / A S q^{1}$ is given the length filtration

$$
F^{n}\left(A / A S q^{1}\right)=\left\langle S q^{1}: I=\left(i_{1}, \cdots, i_{s}\right) \text { admissible, } s \geqq n, i_{s}>1\right\rangle \text {. }
$$


(It follows from this, incidentally, that $E_{0}^{n}\left(A / A S q^{1}\right)$, given the complementary gradation, so $S q^{I}$ occurs in dimension $|I|-n$, is an unstable $A$-module. The same is true of $E^{0}(A)$.) In fact, the filtration on $H^{*}(H)=A / A S q^{1}$ associated with the spectral sequence is precisely the length filtration. To see this we make the following general observation.

For a left $A$-module $M$ let $F^{0} M=M$ and inductively define sub $A$-modules

$$
F^{s+1} M=\left\{S q^{n} x: n+s>|x|, x \in F^{s} M\right\}
$$

For example, on $A / A S q^{1}$ this is precisely the length filtration. In general we call this the unstable filtration, because $E_{0}^{s} M$ (graded by complementary degree) is unstable, and $F^{*} M$ is the largest filtration of $M$ with this property; any other such filtration $\widetilde{F}^{*} M$ has $\widetilde{F}^{s} M \subseteq$ $F^{s} M$ for all $s$. If $M$ is (-1)-connected then $F^{s} M$ is $\left(2^{s}-2\right)$-connected.

Thus in particular the filtration $\widetilde{F}^{*} H^{*}(E)$ associated to the spectral sequence for the spectrum $E$ maps to the unstable filtration. In the example of $A / A S q^{1}$, the quotients agree, so the filtrations agree as claimed.

4.2. The secondary Steenrod action. Consider the filtration induced in stable cohomology $H^{*}(E)$ by the spectral sequence of $\S 1$. Since

$$
S q^{n+1} F^{s} H^{s+t}(E) \subseteq F^{s+1} H^{s+t+n+1}(E)
$$

whenever $n \geqq t$, we may hope to find a representative for $S q^{n+1} \bar{x}$ in $E_{1}$ depending only on a representative for $\bar{x} \in F^{s} H^{s+t}(E)$ in $E_{1}$. In fact we have

THEOREM 4.2.1. Suppose $E$ is a spectrum such that $H_{*}(E)$ is of finite type.

(a) Consider the cohomology spectral sequence with $E_{1}$ obtained from (1.2) with $X(n)=\Omega^{\infty} E(n)$. Let $\bar{x} \in F^{s} H^{s+t}(E)$ and let $n \geqq t$, so that $S q^{n+1} \bar{x} \in F^{s+1} H^{s+t+n+1}(E)$. Let $x_{0} \in \widetilde{H}^{t}\left(E(s)_{0}\right)=E_{1}^{s, t}$ survive to $\bar{x}$. Then

$$
\xi_{11}^{n} \otimes x_{0} \in H^{t+n}\left(\left(\Sigma^{\infty} \Omega^{\infty} E(s)\right)_{0}\right)
$$

maps to an element in $H^{t+n}\left(E(s+1)_{0}\right)$ surviving to $S q^{n+1} \bar{x}$.

(b) Suppose that $Q_{1} H_{*}\left(E_{0}\right)=0$, so that $E_{2}=H\left(L\left(P H^{*}\left(E_{0}\right)\right)\right)$. Let $x_{0} \in L^{s}\left(P H^{*}\left(E_{0}\right)\right)$ survive to $\bar{x} \in F^{s} H^{s+t}(E)$, and let $n \geqq t$. Then $\sigma_{n+1} x \in$ $L^{s+1}\left(P H^{*}\left(E_{0}\right)\right)$ survives to $S q^{n+1} \bar{x}$.

This theorem follows immediately from Lemma 4.2.4 below. 
Let $E$ be a spectrum with $H_{*}(E)$ of finite type, and consider the natural fibration sequence

$$
F \stackrel{j}{\longrightarrow} \Sigma^{\infty} E_{0} \stackrel{k}{\longrightarrow} E \stackrel{i}{\longrightarrow} \Sigma F
$$

on the 0-space level we have a fibration

$$
F_{0} \stackrel{j_{0}}{\longrightarrow} \Omega^{\infty} \Sigma^{\infty} E_{0} \stackrel{k_{0}}{\longrightarrow} E_{0}
$$

Let $x \in H^{t}(E)$ map to $k^{*} x=x_{0} \in H^{t}\left(\Sigma^{\infty} E_{0}\right)=\widetilde{H}^{t}\left(E_{0}\right)$. Let $n \geqq t$, so that $S q^{n+1} x_{0}=0$. Thus there exists $y \in H^{t+n}(F)$ such that $i^{*} y=S q^{n+1} x$. Let $y$ map to $y_{0} \in \widetilde{H}^{t+n}\left(F_{0}\right)$. Then

LEMMA 4.2.4. $\quad j_{0}^{*}\left(\xi_{11}^{n} \otimes x_{0}\right)=y_{0}$ •

Proof. Let $K=\left\{K_{t}\right\}$ denote the mod 2 Eilenberg-MacLane spectrum, and let

$$
L \longrightarrow K \stackrel{S q^{n+1}}{\longrightarrow} \Sigma^{n+1} K
$$

be a fibration sequence in $\mathscr{L}$. Let $\iota_{t+n} \in H^{t+n}\left(K_{t+n}\right)$ be the fundamental class, and let $\bar{\iota}_{t+n} \in H^{t+n}\left(L_{t}\right)$ map to $\iota_{t+n}$ under the boundary homomorphism. Then the reduced coaction $\bar{\psi}: H^{*}\left(L_{t}\right) \rightarrow \hat{R} \otimes H^{*}\left(L_{t}\right)$ satisfies

$$
\bar{\psi} \bar{\iota}_{t+n}=\xi_{11}^{n} \otimes \iota_{t}
$$

This formula is just dual to the well known fact [3] that the fundamental homology classes in $L_{t}$ are related by $Q^{n}$.

Now, since $k^{*} x=x_{0}$ has $S q^{n+1} x_{0}=0$, we have maps $y$ and $z$ in the commutave diagram of spectra

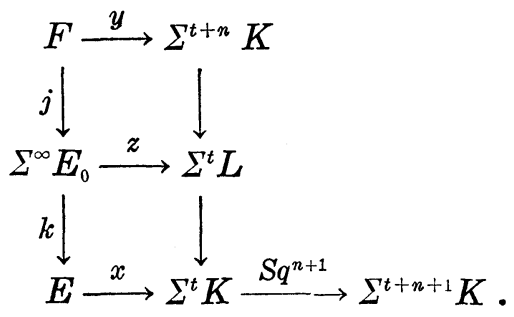

At the level of 0 -spaces, we have a commutative diagram 




where $\beta=\Omega^{\infty} \beta_{\Sigma^{\infty} E_{0}}^{\infty}, \alpha=\alpha_{\Omega^{\infty} \Sigma^{\infty} E_{0}^{\prime}}^{\infty}$, and $\varphi=\Omega^{\infty} \beta_{\Sigma^{\prime} t_{L}}^{\infty}$. Chasing the element $\bar{\iota}_{n+t} \in H^{n+t}\left(L_{t}\right)$ and using (4.2.5), we have

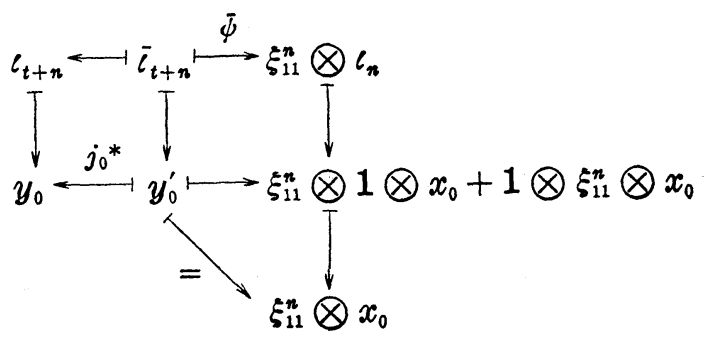

so the result follows.

EXAMPLE 4.2.6. Returning again to the case of the integral Eilenberg-MacLane spectrum, we see that Theorem 4.2.1 forces the spectral sequence to collapse. Thus we have recovered the Steenrod algebra (additively) using "only" the structure of $H_{*}\left(\Omega^{\infty} \Sigma^{\infty} X\right)$ and the existence of a map $S q^{n+1}$ satisfying (4.2.5).

\section{REFERENCES}

1. A. K. Bousfield and E. B. Curtis, A spectral sequence for the homotopy of nice spaces, Trans. Amer. Math. Soc., 151 (1970), 457-479.

2. A. K. Bousfield and D. M. Kan, The homotopy spectral sequence of a space with coefficients in a ring, Topology, 11 (1972), 79-106.

3. E. H. Brown and S. Gitler, A spectrum whose cohomology is a certain cyclic module over the Steenrod algebra, Topology, 12 (1973), 283-295.

4. F. R. Cohen, T. J. Lada, and J. P. May, The Homology of Iterated Loop Spaces, Springer-Verlag Lecture Notes in Mathematics 533, (1976).

5. K. V. A. M. Gugenheim, On extension of algebras, coalgebras and Hopf algebras I, Amer. J. Math., 84 (1962), 349-385.

6. D. Husemoller and J. C. Moore, Differential graded homological algebra of several variables, Symp. Math., IV (1970), Instituto Nazionale di Alta Mat., 397-429.

7. I. Madsen, On the action of the Dyer-Lashof algebra in $H_{*} G$, Pacific J. Math., 60 (1975), 235-275.

8. J. P. May, Categories of spectra and infinite loop spaces, Springer-Verlag Lecture Notes in Mathematics, 99 (1969), 448-479.

9. J. C. Moore and L. Smith, Hopf algebras and multiplicative fibrations II, Amer. J. Math., 90 (1968), 1113-1150. 
10. S. B. Priddy, Koszul resolutions, Trans. Amer. Math. Soc., 152 (1970), 39-60.

11. W. M. Singer, The algebraic EHP sequence, Trans. Amer. Math. Soc., 201 (1975), $367-382$.

Received October 24, 1977. Supported in part by NSF.

HARVARD UNIVERSITY

CAMBRIDGE, MA 02138 



\section{PACIFIC JOURNAL OF MATHEMATICS}

EDITORS

RICHARD ARENS (Managing Editor)

University of California

Los Angeles, California 90024

C. W. Curtis

University of Oregon

Eugene, OR 97403

C. C. MOORE

University of California

Berkeley, CA 94720
J. DUGUNDJI

Department of Mathematics University of Southern Californı Los Angeles, California 90007

R. Finn and J. Milgram Stanford University Stanford, California 94305

\section{ASSOCIATE EDITORS}

E. F. BECKENBACH

B. H. NEUMANN

F. WOLF

K. YoSHIDA

\section{SUPPORTING INSTITUTIONS}

UNIVERSITY OF BRITISH COLUMBIA
CALIFORNIA INSTITUTE OF TECHNOLOGY
UNIVERSITY OF CALIFORNIA
MONTANA STATE UNIVERSITY
UNIVERSITY OF NEVADA, RENO
NEW MEXICO STATE UNIVERSITY
OREGON STATE UNIVERSITY
UNIVERSITY OF OREGON

UNIVERSITY OF SOUTHERNEALIFORNIA STANFORD UNIVERSITY UNIVERSITY OF HAWAII UNIVERSITY OF TOKYO UNIVERSITY OF UTAH WASHINGTON STATE UNIVERSITY UNIVERSITY OF WASHINGTON 


\section{Pacific Journal of Mathematics \\ Vol. 79 , No. 1 \\ May, 1978}

Teófilo Abuabara, A remark on infinitely nuclearly differentiable

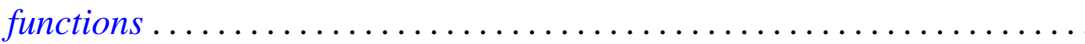

David Fenimore Anderson, Projective modules over subrings of $k[X, Y]$

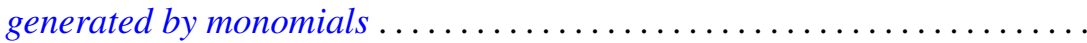

Joseph Barback and Thomas Graham McLaughlin, On the intersection of

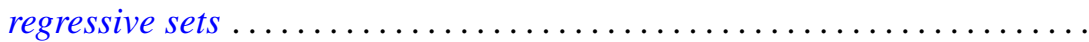

Murray Bell, John Norman Ginsburg and R. Grant Woods, Cardinal inequalities for topological spaces involving the weak Lindelof



Laurence Richard Boxer, The space of ANRs of a closed surface ............

Zvonko Cerin, Homotopy properties of locally compact spaces at

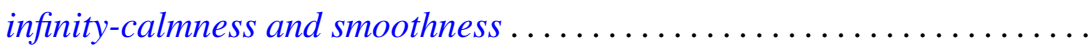

Isidor Fleischer and Ivo G. Rosenberg, The Galois connection between partial functions and relations..................................

John R. Giles, David Allan Gregory and Brailey Sims, Geometrical implications of upper semi-continuity of the duality mapping on a Banach



Troy Lee Hicks, Fixed-point theorems in locally convex spaces ............ Hugo Junghenn, Almost periodic functions on semidirect products of transformation semigroups ........................

Victor Kaftal, On the theory of compact operators in von Neumann algebras. II . . . .

Haynes Miller, A spectral sequence for the homology of an infinite

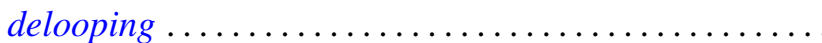

Sanford S. Miller, Petru T. Mocanu and Maxwell O. Reade, Starlike integral operators...

Stanley Stephen Page, Regular FPF rings ...............

Ghan Shyam Pandey, Multipliers for C, 1 summability of Fourier series ...

Shigeo Segawa, Bounded analytic functions on unbounded covering surfaces...

Steven Eugene Shreve, Probability measures and the C-sets of

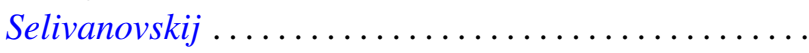

Tor Skjelbred, Combinatorial geometry and actions of compact Lie groups....

Alan Sloan, A note on exponentials of distributions.

Colin Eric Sutherland, Type analysis of the regular representation of a nonunimodular group.

Mark Phillip Thomas, Algebra homomorphisms and the functional

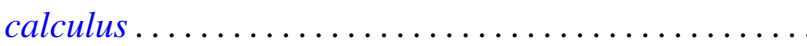

Sergio Eduardo Zarantonello, A representation of $H^{p}$-functions with

$0<p<\infty$. 